\title{
Temporary Inactivation of the Bed Nucleus of the Stria Terminalis But Not of the Amygdala Blocks Freezing Induced by Trimethylthiazoline, a Component of Fox Feces
}

\author{
Markus Fendt, Thomas Endres, and Raimund Apfelbach \\ Tierphysiologie, Universität Tübingen, D-72076 Tübingen, Germany
}

\begin{abstract}
Presentation of trimethylthiazoline (TMT, a component of fox feces) to laboratory rats elicits freezing, a prominent behavioral sign of anxiety or fear. The present study investigated the neural basis of this unlearned response. Muscimol, a $\mathrm{GABA}_{\mathrm{A}}$ receptor agonist, was injected $(4.4 \mathrm{nmol} / 0.5 \mu \mathrm{l})$ into the bed nucleus of the stria terminalis (BNST) as well as into the amygdala, two brain areas known to be involved in anxiety and fear. Temporary inactivation of the BNST but not of the amygdala significantly blocked TMT-induced freezing. This effect was not caused by an enhancement of motor activity after BNST inactivation. In addition, these results confirm previous studies showing that freezing is possible despite amygdala inactivation. These results, and other findings in the literature, suggest that the BNST is critically involved in unlearned fear, whereas the amygdala is more involved in the acquisition and expression of learned fear.
\end{abstract}

Key words: amygdala; bed nucleus of the stria terminalis; fear; odor; muscimol; predator; temporary lesion

\section{Introduction}

Fear is a functional behavioral system that prepares an animal for a fast and effective hide, flight, or fight response in the presence of potentially dangerous environmental threats (Fanselow, 1991; Fendt and Fanselow, 1999). These responses are critical for the survival of small vertebrates such as rats because of the large predator pressure under which they live. When a rat is attacked by a predator in the wild, there is not much time for defensive maneuvers (Bolles, 1970), so an effective elicitation of these defensive behaviors by signals predicting predators is a valuable evolutionary benefit (also see Downes, 2002). Signals predicting a potential predator may be innately recognized or learned through experience (Blanchard and Blanchard, 1971, 1972).

One ecologically relevant signal that may elicit defensive behaviors, and fear, is the odor of a predator. Indeed, research with rats and mice has shown that fear is elicited by the odor of cats (Blanchard and Blanchard, 1989; Zangrossi and File, 1992) or foxes (Wallace and Rosen, 2000; Perrot-Sinal et al., 2000). Mice also exhibit fear in the presence of other predators (e.g., snake odor) (Dell'Omo and Alleva, 1994). Long-lasting exposure to predator odors has also been shown to affect the hormonal state, fertility, and reproduction success of animals (Vasilieva et al., 2000; Apfelbach et al., 2001). In most of the experiments cited above, the complete odor of a predator was used. In some cases, the specific component of the predator odor responsible for elicitation of fear-related behavior and concomitant physiological

\footnotetext{
Received July 24, 2002; revised 0ct. 8, 2002; accepted 0ct. 22, 2002.

This research was supported by Deutsche Forschungsgemeinschaft Grant SFB 550/C8. We are grateful to Christine Buck and David Hamm for their help in collecting the data, Helga Zillus for excellent technical assistance, and Dr. Rick Richardson for helpful comments regarding this manuscript.

Correspondence should be addressed to Dr. Markus Fendt, Tierphysiologie, Universität Tübingen, Auf der Morgenstelle 28, D-72076 Tübingen, Germany. E-mail: markus.fendt@uni-tuebingen.de.

Copyright $\odot 2002$ Society for Neuroscience $\quad 0270-6474 / 02 / 220023-06 \$ 15.00 / 0$
}

changes is known. For example, the critical component of the odor of fox feces is trimethylthiazoline (TMT). TMT is very effective in eliciting fear: Wild rats showed an avoidance response of TMT, although they were naive to foxes (Vernet-Maury et al., 1984). Similarly, Wallace and Rosen $(2000,2001)$ demonstrated that naive laboratory rats also were fearful, as shown by freezing, in the presence of TMT.

Freezing is one of the most prominent behavioral symptoms of fear in rats (Griffith, 1920; Blanchard and Blanchard, 1971; Bolles and Collier, 1976). During freezing, only movements associated with respiration are observable. The biological significance of freezing might be to reduce movements that would increase the rat's detectability to predators (Fanselow and Lester, 1988). In any case, a number of studies have investigated the neural basis of fear-related behaviors such as freezing (Fendt and Fanselow, 1999; LeDoux, 2000; Walker and Davis, 2002). These studies have established a brain circuitry including the amygdala, the bed nucleus of the stria terminalis (BNST), the periaqueductal gray, as well as several other nuclei, as being responsible for the expression of learned and unlearned fear responses.

Wallace and Rosen (2001) demonstrated that neurotoxic lesions of the amygdala did not affect TMT-induced freezing. This finding suggests that other parts of the brain circuitry described above must be responsible for the elicitation of freezing by TMT. In that regard, it has been shown that the unconditioned enhancement of the startle response by bright light is mediated by the BNST (Walker and Davis, 1997). In this study it was suggested that the BNST may mediate unlearned fear (such as rats' anxiety during bright light), whereas the amygdala may mediate learned (conditioned) fear. Because the fear elicited by predator odors is not learned (the rats have never had any experience with foxes), we suggest that the BNST might mediate freezing elicited by TMT. 
The present study directly addressed this hypothesis. Specifically, we induced temporary lesions of the BNST or the amygdala by local microinjections of the $\mathrm{GABA}_{\mathrm{A}}$ receptor agonist muscimol and then measured TMT-induced freezing. In a post hoc experiment, possible effects of muscimol injections into the BNST on motor activity in an open field were investigated. This experiment was done to exclude the possibility that the observed effect of BNST inactivation in the main experiment was an artifact of the effects of muscimol on motor activity.

\section{Materials and Methods}

Subjects. Thirty-eight male Sprague Dawley rats (Charles River, Sulzfeld, Germany) weighing 220-260 gm at the time of the surgery were used. The animals were maintained on a $12 \mathrm{hr}$ light/dark cycle, and food and water were available ad libitum. All experiments were performed in accordance with ethical guidelines for the use of animals in experiments and were approved by the local animal care committee (Regierungspräsidium Tübingen, ZP 5/99).

Surgery. Rats were anesthetized with ketamine/xylazine (9:1; $100 \mathrm{mg} /$ $\mathrm{kg}$, i.p.) and placed in a stereotaxic frame with blunt ear bars. Two stainless guide cannulas (diameter, $0.7 \mathrm{~mm}$ ) were implanted bilaterally into the brain aiming at the BNST $(0.5 \mathrm{~mm}$ rostral, $\pm 1.4 \mathrm{~mm}$ lateral, $6.5 \mathrm{~mm}$ ventral to bregma) (Paxinos and Watson, 1997) or the amygdala $(2.8 \mathrm{~mm}$ caudal, $\pm 5.0 \mathrm{~mm}$ lateral, $7.0 \mathrm{~mm}$ ventral to bregma) (Paxinos and Watson, 1997). The cannulas were fixed to the skull with dental cement and three anchoring screws. After surgery and between the tests, the cannulas were fitted with stylets (diameter, $0.4 \mathrm{~mm}$ ) to maintain patency. Rats were given $4-6 \mathrm{~d}$ to recover from surgery before testing.

Apparatus for odor exposure. Rats $(n=28)$ were placed in one of three identical exposure boxes $(30 \times 30 \times 30 \mathrm{~cm})$ made of polyvinyl chloride to assess TMT-elicited freezing. The front doors of these chambers were constructed of Plexiglas to permit observation of the rats. The behavior of the animals was recorded for later analyses.

Each exposure box was connected via Teflon tubing to a generator supplying charcoal filtered air; the outflow of the box was connected to an exhaust system. The air stream could be directed by electrically operated three-way Teflon valves either directly to the exposure boxes or through a glass bottle containing the odorant [20 $\mu$ l of 2,4,5trimethylthiazoline (PheroTech Inc., Delta, Canada) on a piece of filter paper] and then to the boxes. In both cases, clean air or air/odor flows were regulated with needle valves $(17 \mathrm{l} / \mathrm{min})$ and monitored by flow meters.

Procedure for odor exposure. To familiarize the rats with the olfactory exposure boxes, each animal was placed for $10 \mathrm{~min}$ into one of the exposure boxes (once per day on 5 consecutive days). On the following day, freezing behavior of the animals in the boxes was recorded during a 15 min period. After the fourth minute, the valve controlling the air flow was switched, but clean air (i.e., no TMT) was still presented to the animals on this first test day. On the next $2 \mathrm{~d}$, each animal received (in a pseudorandomized manner) bilateral injections of either $4.4 \mathrm{nmol}$ of muscimol [dissolved in $0.5 \mu \mathrm{l}$ of artificial CSF (ACSF)] or ACSF alone into the BNST $(n=12)$ or amygdala $(n=13)$. The solutions were infused bilaterally at a rate of $0.1 \mu \mathrm{l} / 10 \mathrm{sec}$. After the injection, cannulas were left in place for another $2 \mathrm{~min}$ to allow diffusion of the solution away from the cannula. Previous research has shown that the injected drug diffuses in an area of $\sim 1 \mathrm{~mm}$ diameter around the injection site with these sorts of parameters (Miserendino et al., 1990).

Immediately after the infusions, animals were placed into the odor exposure boxes and were observed for $15 \mathrm{~min}$. After $4 \mathrm{~min}$, the Teflon valves were switched and TMT was directed into the exposure boxes. After this test, animals were placed into a cage located in a fume hood for $2 \mathrm{hr}$ and then returned to their home cages. After each experimental session, the odor chamber, and tubing, was thoroughly washed with $70 \%$ ethanol and ventilated with clean air for $2 \mathrm{hr}$.

The videotapes from all experiments were analyzed by one observer who was not aware of the animal's condition. Freezing was used to measure fear. Freezing is characterized by crouching, with cessation of move- ments except those associated with breathing (cf. Blanchard and Blanchard, 1969). The percentage of time spent freezing was calculated for each rat for every minute for each test session [(seconds of freezing/60 sec) $\times 100]$.

Motor activity in the open field. Motor activity of rats $(n=10)$ was measured in an open field made of gray plastic with a diameter of $80 \mathrm{~cm}$ (height of the walls, $25 \mathrm{~cm}$ ). The open field was divided into 16 subfields. After injections (as above), each rat was placed in the center of the open field, and horizontal motor activity (line crossings of all four legs) was quantified over $15 \mathrm{~min}$. Line crossings were evaluated on-line by an observer via a video camera.

Statistical analysis. Statistical analysis of the freezing data were accomplished by ANOVA (followed by post hoc Tukey tests). Motor activity data, for groups and across time, were analyzed by ANOVA and by $t$ tests.

\section{Results}

Histology

The injection sites within the BNST and the amygdala are shown in Figure 1. For those rats tested for TMT-induced freezing, 12 injection sites were located in the BNST and 13 injection sites were located in the lateral nucleus of the amygdala. The data from three animals were excluded from analysis because of misplaced injection sites (hippocampus, putamen caudatus). For those rats tested for motor activity in the open field, all 10 injection sites were located in the BNST.

\section{TMT-induced freezing}

Analysis of freezing during the first test day (clean air only) revealed no effects of group, time, or interaction ( $F$ values of $<1.53)$. Levels of freezing were consistently low in this test (19.524.1\%; data not shown).

\section{ACSF-injected rats}

To determine the effects of TMT on freezing, two sets of withinsubjects analyses were done. In the first set, performance during odor presentation was compared with baseline performance. As in previous studies (Wallace and Rosen, 2000, 2001), TMT induced freezing in ACSF-infused rats (Figs. 2, open symbols, 3, ACSF condition; $F$ values $=21.3 ; p$ values of $<0.001$ ). Post hoc comparisons between freezing rates of the first $4 \mathrm{~min}$ (no TMT) and the freezing rates during TMT presentation revealed a significant increase in freezing during TMT presentation ( $p$ values of $<0.001)$. No differences in TMT-induced freezing were found between amygdala- and BNST-injected rats (interaction of TMT condition and nucleus: $F<1.0$ ).

To ensure that these increases in freezing to the odor were not merely attributable to the passage of time, a second set of analyses was done: Freezing during TMT presentation was significantly higher than freezing with clean air during the same time window of the test on the previous day ( $p$ values of $<0.001$ ).

\section{Injections of muscimol into the amygdala}

To estimate the role of the amygdala and BNST in TMT-induced freezing, the freezing rates after muscimol injections were compared with those after ACSF injections. Muscimol injections into the amygdala did not affect TMT-induced freezing (Figs. 2, 3; interaction of drug and odor, $F<1.0$ ). This was confirmed by post hoc comparison showing an increase in freezing rate during TMT presentation $(p<0.001)$.

Injections of muscimol into the BNST

In contrast, muscimol injected into the BNST blocked the increase in freezing elicited by TMT (Figs. 2, 3; interaction of drug and odor: $\left.F_{(1,30)}=4.98 ; p=0.03\right)$. This was confirmed by a post hoc Tukey test showing no differences in freezing during the base- 

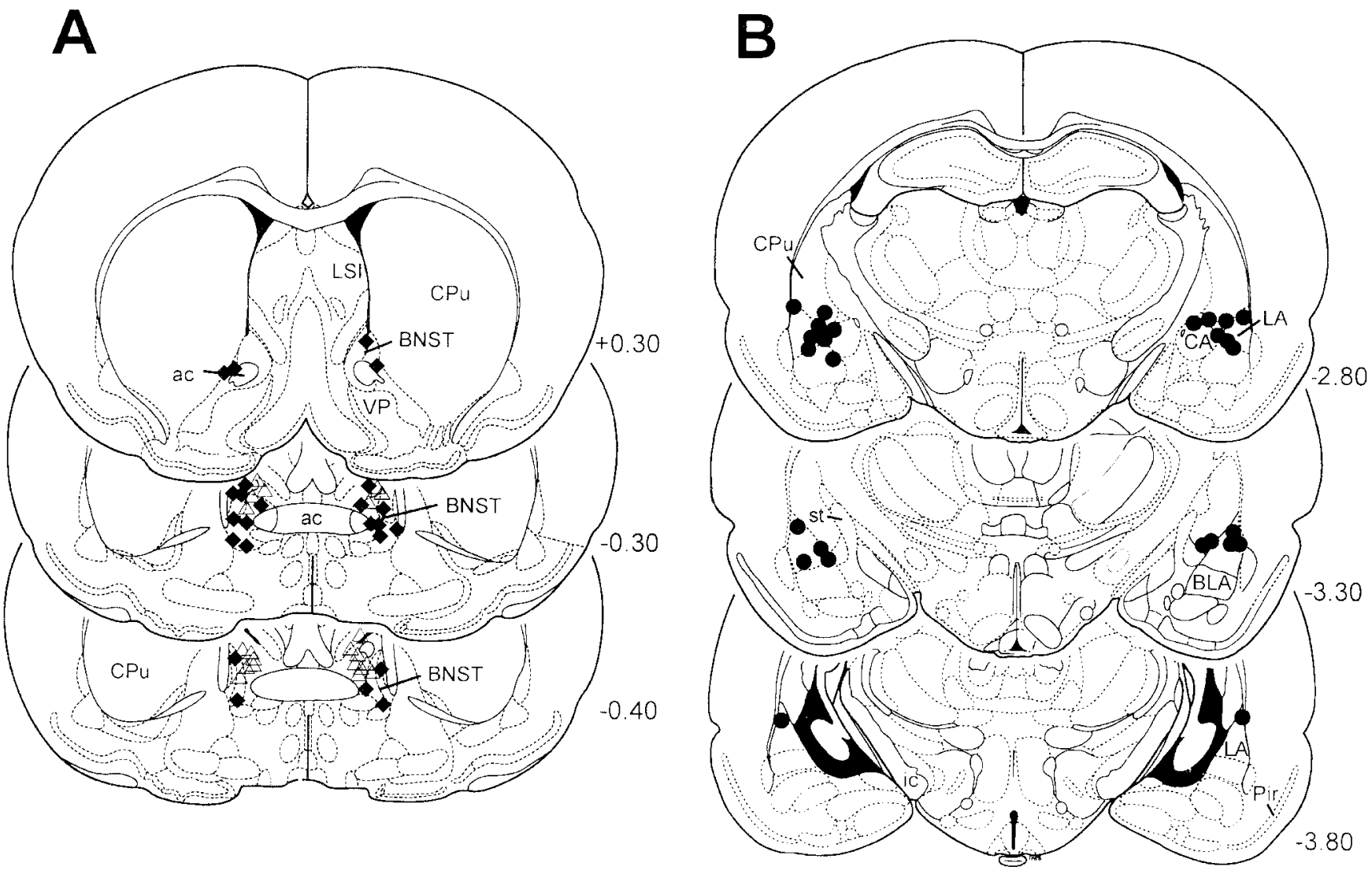

Figure 1. Reconstructions of the different injection sites of ACSF and muscimol into the BNST $(A, \bullet$, test on TMT-induced freezing; $\triangle$, test on motor activity) and amygdala ( $B)$. The coronal sections were taken from the atlas of Paxinos and Watson (1997); numbers to the right indicate distance (in millimeters) from bregma. ac, Anterior commissure; $B L A$, basolateral nucleus of the amygdala; $C A$, central nucleus of the amygdala; $C P u$, caudate putamen; $i$, internal capsule; $L A$, lateral nucleus of the amygdala; $L S I$, lateral septal nucleus, intermediate part; $P i r$, piriform cortex; $s t$, stria terminalis; $V P$, ventral pallidum.

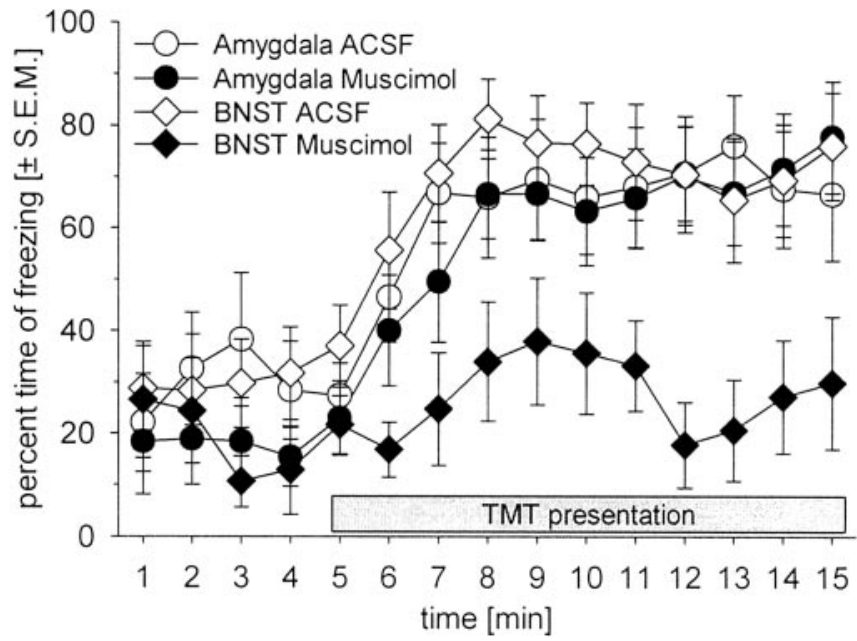

Figure 2. Time courses of the mean \pm SEM percentage of time spent freezing to TMT in BNST-injected (circles) and amygdala-injected (diamonds) animals. The gray box at the bottom indicates the duration of TMT presentation.

line period and during TMT presentation after muscimol injections into the BNST ( $p=0.81$ ). It is important to note that the baseline freezing response before TMT presentation was not affected by muscimol injections into either the amygdala or the BNST (post hoc Tukey tests: comparisons between ACSF and muscimol; $p$ values $>0.43)$.

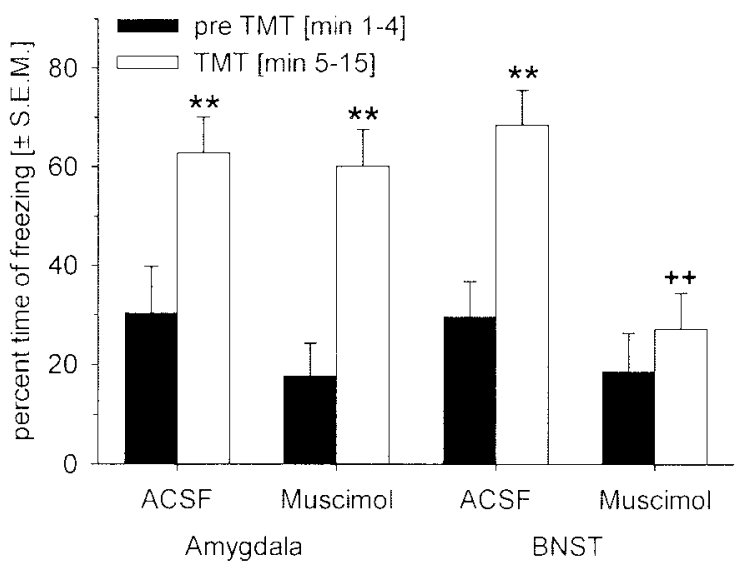

Figure 3. Mean \pm SEM percentage of time spent freezing to TMT in the pre-odor ( $\min 1-4)$ and post-odor (min 5-15) condition. ${ }^{* *} p<0.01$ compared with the pre-TMT condition; ${ }^{++} p<0.01$ compared with ACSF treatment (ANOVA followed by a post hoc Tukey test).

\section{Motor activity in the open field}

To ensure that the blockade of TMT-induced freezing after muscimol injections into the BNST was not caused by a simple enhancement of motor activity, we measured the effects of muscimol injections into the BNST on horizontal motor activity in the open field. The results showed that motor activity in the open field was not affected by muscimol injections into the BNST (Fig. 4 ; effect of drug and interaction drug $\times$ time: $F$ values of $<1.5 ; p$ 


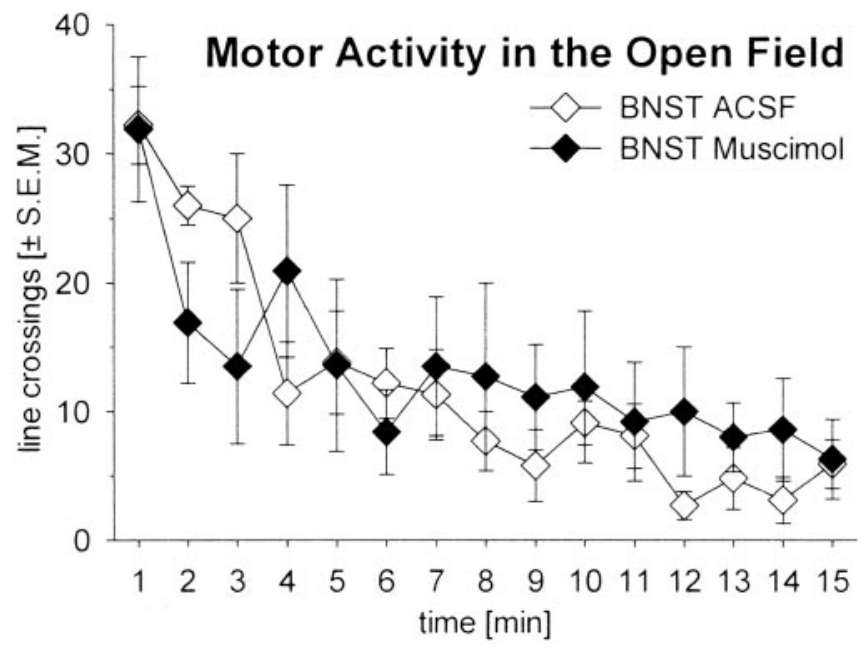

Figure 4. Time course of motor activity measured by line crossings \pm SEM during a $15 \mathrm{~min}$ period in an open field. The rats received injections of ACSF $(\diamond)$ or muscimol $(\diamond)$ into the BNST.

values of $<0.15)$. A comparison of the total motor activity collapsed over the $15 \mathrm{~min}$ test also failed to reveal a significant group difference (Student's $t$ test; $t=0.28 ; p=0.78$ ).

\section{Discussion}

The present study tested whether the BNST and the amygdala are involved in TMT-elicited freezing. Specifically, we temporarily inactivated the BNST and the amygdala by local microinjections of the $\mathrm{GABA}_{\mathrm{A}}$ receptor agonist muscimol and then measured TMT-induced freezing. Most importantly, the present study demonstrated a total disruption of TMT-induced freezing after temporary inactivation of the BNST but not of the amygdala. This effect was not attributable to an increase in motor activity after muscimol injections into the BNST.

\section{TMT-induced freezing}

In the present study, TMT induced a high rate of freezing. Similar results were reported by Wallace and Rosen (2000, 2001). Yet McGregor et al. (2002), who directly compared the effects of TMT and cat odor on the behavior of rats, questioned whether TMT induces a predator-specific fear response in rats. They observed species-specific defensive responses such as concealing and head-out behavior to cat odor but not to TMT.

A comparison of complete odors (e.g., via a cat collar) and individual components of an odor (e.g., TMT) should be done with caution. Furthermore, it should be noted that there were some methodological differences between the different cited studies: In all experiments, test chambers of similar sizes were used, but those used by McGregor et al. (2002) provided the rat with the opportunity to hide. In the study by Wallace and Rosen (2000, 2001), as well as in our study, hiding was not possible. Blanchard and Blanchard (1990) showed that in rats, the quality of defense behavior depends on the possibility of flight or hiding. In our study, the rats did not have any possibility to flee or to hide. Therefore, the only possible useful defense behavior was freezing, and the incidence of freezing was substantially increased by TMT presentation.

Before TMT presentation, the animals in the present study showed a freezing rate between 20 and $40 \%$. This is a higher rate than observed by Wallace and Rosen (2000) who reported a baseline freezing response between 10 and $20 \%$, or in the studies of the
Fanselow group, who reported a baseline freezing response of almost 0\% (Maren et al., 1997; Anagnostaras et al., 1999). These differences in baseline freezing are likely caused by slight differences in the testing procedures or the test chambers. In any case, the high rate of baseline freezing observed in this study increases the likelihood of detecting any motor-enhancing effects of muscimol.

\section{Role of the amygdala in TMT-induced freezing}

The present study clearly shows that temporary inactivation of the amygdala does not affect TMT-induced freezing. This supports the results of Wallace and Rosen (2001) demonstrating an unimpaired freezing response to TMT after neurotoxic lesions of the amygdala. In that study, electrolytic lesions of the amygdala reduced or blocked TMT-induced freezing. The authors explained these apparently contradictory findings by suggesting that electrolytic lesions also destroy fibers of passage, and that this was responsible for the observed impairment of TMT-induced freezing. This sort of rationale could also explain the impairment of freezing to cat odor that has been found after chemical or radiofrequency lesions of the amygdala (Blanchard and Blanchard, 1972; Fox and Sorenson, 1994). In our study, we temporarily inactivated amygdaloid neurons (especially in the central and lateral part of the amygdala) by local microinjections of muscimol. Because muscimol works via $\mathrm{GABA}_{\mathrm{A}}$ receptors, fibers of passage are not inactivated. Therefore, the findings of the present study are in agreement with the explanation offered by Wallace and Rosen (2001). In addition, our study confirmed findings (Wallace and Rosen, 2001) that the lateral nucleus of the amygdala is not necessary for the production of freezing per se.

This finding might be seen as surprising given that the amygdala is one of the main neural structures involved in behavioral fear responses. Different studies have demonstrated that the amygdala, especially its lateral, basolateral, and central nuclei, is necessary for the acquisition and expression of conditioned fear (Davis et al., 1993; Fendt and Fanselow, 1999; LeDoux, 2000). Davis and colleagues (Davis, 1996; Walker and Davis, 1997; Davis and Shi, 1999) reported that the central part of the amygdala is not involved in unlearned fear. In contrast, the basolateral part of the amygdala mediates both learned and unlearned fear. It should be noted that the results presented here only partly confirm these results. Specifically, our injection sites were located in the dorsal part of the amygdaloid complex, primarily in the central or lateral part of the amygdala. Muscimol injections into all of these sites did not block TMT-induced freezing, whereas in the study by Walker and Davis (1997), only injections into the central nucleus of the amygdala did not affect unlearned fear. We suggest that the different subnuclei of the amygdala may be differently involved in the mediation of unlearned fear depending on the modality of the eliciting stimulus. For example, the anterior cortical nucleus of the amygdala receives strong input from the olfactory system (Shipley et al., 1995), and the medial nucleus of the amygdala shows a large increase in c-fos activity after presentation of cat odor (Dielenberg et al., 2001). In addition, there was no increase in c-fos activity within the central and the basolateral nuclei of the amygdala after presentation of cat odor (Dielenberg et al., 2001).

Both the anterior cortical and the medial nucleus of the amygdala are far away $(>2 \mathrm{~mm})$ from our injection sites, and it is questionable whether muscimol injected in the present study reached these nuclei. However, the ineffective injection sites into the dorsal part of the amygdala are closer to these nuclei than the effective injection sites into the BNST, so this provides a strong argument against the idea that the effect of muscimol within the BNST was caused by diffusion to these nuclei. 
The present study investigated the role of the amygdala and the BNST in freezing elicited by an unlearned olfactory stimulus. Several recent studies have used an odor as a conditioned stimulus (CS) in a fear-conditioning paradigm (Otto et al., 1997; Richardson et al., 1999; Paschall and Davis, 2002). It has been shown that olfactory fear conditioning is mediated by the basolateral nucleus of the amygdala (Cousens and Otto, 1998). Therefore, we suggest that the amygdala would also be necessary for the learning of an association between a CS and TMT. This idea is supported by electrophysiological studies showing that neurons with multimodal input (e.g., visual and olfactory) are located in the more dorsal parts of the amygdala, whereas neurons with unimodal olfactory input are located in the more ventral parts of the amygdala (Uwano et al., 1995).

\section{Role of the BNST in TMT-induced freezing}

In the present study, temporary inactivation of the BNST completely blocked TMT-induced freezing. It is important to note that this blockade of freezing was not a side effect of muscimol injections into the BNST on motor activity. Our control experiment demonstrated no changes in motor activity after muscimol injections into the BNST for $15 \mathrm{~min}$ (the duration of the TMT tests). To the best of our knowledge, this is the first study showing that the BNST is involved in olfactory-induced fear behavior. Nevertheless, this finding is not surprising because (1) the BNST is strongly connected to the olfactory system (Shipley et al., 1995) and (2) the BNST is involved in the mediation of stress (Pacak et al., 1995) and unconditioned fear responses (Walker and Davis, 1997; Davis and Shi, 1999). Walker and Davis (1997) blocked glutamate receptors within the BNST and observed a disruption of light-enhanced startle but not of conditioned fear-potentiated startle. Because bright light is an unlearned aversive stimulus, they suggested that the BNST is more involved in unconditioned fear (anxiety), whereas the amygdala is more involved in learned fear. This idea is supported by the results of the present study.

Both the amygdala and the BNST have very similar afferent connections and projections to various brain regions known to be involved in behavioral and autonomic symptoms of fear and anxiety (Davis and Shi, 1999). Our results strongly confirm the hypothesis that the BNST is a crucial brain nucleus in the mediation of unlearned fear; TMT is an unlearned olfactory stimulus, and the fear response to TMT was completely blocked by BNST inactivation. Additional studies will investigate whether the effects of other predator odors [and other unlearned fear-eliciting stimuli (e.g., owl calls)] (Hendrie, 1991) are also mediated by the BNST.

\section{Conclusion}

In summary, we demonstrated that TMT, a component of fox feces, is able to elicit freezing in rats, and that the TMT-induced freezing response can be blocked by temporary inactivation of the BNST but not of the amygdala. These results confirm the hypothesis that the BNST is important for the mediation of unlearned fear responses, whereas the amygdala is more involved in the learning of fear and in responses to conditioned stimuli.

\section{References}

Anagnostaras SG, Maren S, Fanselow MS (1999) Temporally graded retrograde amnesia of contextual fear after hippocampal damage in rats: within-examination. J Neurosci 19:1106-1114.

Apfelbach R, Wiest H, Vasilieva NY (2001) Ferret (Mustela putorius f. furo) odor affects the estrous cycle in Campell's hamster females (Phodopus campbelli). Wiss Mitt Niederösterr Landesmuseum 14:147-152.

Blanchard DC, Blanchard RJ (1972) Innate and conditioned reactions to threat in rats with amygdaloid lesions. J Comp Physiol Psychol 81:281-290.
Blanchard RJ, Blanchard DC (1969) Crouching as an index of fear. J Comp Physiol Psychol 67:370-375.

Blanchard RJ, Blanchard DC (1971) Defensive reactions in the albino rat. Learn Motiv 2:351-362.

Blanchard RJ, Blanchard DC (1989) Antipredator defensive behaviors in a visible burrow system. J Comp Psychol 103:70-82.

Blanchard RJ, Blanchard DC (1990) An ethoexperimental analysis of defense, fear, and anxiety. In: Anxiety (McNaughton N, Andrews G, eds), pp 124-133. Dunedin, New Zealand: University of Otago.

Bolles RC (1970) Species-specific defensive reactions and avoidance learning. Psychol Rev 71:32-48.

Bolles RC, Collier AC (1976) Effect of predictive cues on freezing in rats. Anim Learn Behav 4:6-8.

Cousens G, Otto T (1998) Both pre- and posttraining excitotoxic lesions of the basolateral amygdala abolish the expression of olfactory and contextual fear conditioning. Behav Neurosci 112:1092-1103.

Davis M (1996) Differential roles of the amygdala and bed nucleus of the stria terminalis in conditioned fear and startle enhanced by corticotropinreleasing hormone. In: Perception, memory, and emotion: frontiers in neuroscience (Ono T, McNaughton BL, Molotchnikoff S, Rolls ET, Nishijo H, eds), pp 525-548. Oxford: Elsevier.

Davis M, Shi C (1999) The extended amygdala: are the central nucleus of the amygdala and the bed nucleus of the stria terminalis differentially involved in fear versus anxiety. Ann NY Acad Sci 877:281-291.

Davis M, Falls WA, Campeau S, Kim M (1993) Fear-potentiated startle: a neural and pharmacological analysis. Behav Brain Res 58:175-198.

Dell'Omo G, Alleva E (1994) Snake odor alters behavior, but not pain sensitivity in mice. Physiol Behav 55:125-128.

Dielenberg RA, Hunt GE, McGregor IS (2001) "When a rat smells a cat": the distribution of Fos immunoreactivity in rat brain following exposure to a predatory odor. Neuroscience 104:1085-1097.

Downes SJ (2002) Does responsiveness to predator scents affect lizard survivorship? Behav Ecol Sociobiol 52:38-42.

Fanselow MS (1991) The midbrain periaqueductal gray as a coordinator of action in response to fear and anxiety. In: The midbrain periaqueductal gray matter (Depaulis A, Bandler R, eds), pp 151-173. New York: Plenum.

Fanselow MS, Lester LS (1988) A functional behavioristic approach to aversively motivated behavior: predatory imminence as a determinant of the topography of defensive behavior. In: Evolution and learning (Bolles RC, Beecher MD, eds), pp 185-211. New York: Hillsdale.

Fendt M, Fanselow MS (1999) The neuroanatomical and neurochemical basis of conditioned fear. Neurosci Biobehav Rev 23:743-760.

Fox RJ, Sorenson CA (1994) Bilateral lesions of the amygdala attenuate analgesia induced by diverse environmental challenges. Brain Res 648:215-221.

Griffith CR (1920) The behavior of white rats in the presence of cats. Psychobiology 2:19-28.

Hendrie CA (1991) The calls of murine predators activate endogenous analgesia mechanisms in laboratory mice. Physiol Behav 49:569-573.

LeDoux JE (2000) Emotion circuits in the brain. Annu Rev Neurosci 23:155-184.

Maren S, Aharanov G, Fanselow MS (1997) Neurotoxic lesions of the dorsal hippocampus and Pavlovian fear conditioning in rats. Behav Brain Res $88: 261-274$.

McGregor IS, Schrama L, Ambernoon P, Dielenberg RA (2002) Not all "predator odours" are equal: cat odour but not 2,4,5 trimethylthiazoline (TMT; fox odour) elicits specific defensive behaviours in rats. Behav Brain Res 129:1-16.

Miserendino MJD, Sananes CB, Melia KR, Davis M (1990) Blocking of acquisition but not expression of conditioned fear-potentiated startle by NMDA antagonists in the amygdala. Nature 345:716-718.

Otto T, Cousens G, Rajewski K (1997) Odor-guided fear conditioning in rats. 1. Acquisition, retention, and latent inhibition. Behav Neurosci 111:1257-1264.

Pacak K, McCarty R, Palkovits M, Kopin IJ, Goldstein DS (1995) Effects of immobilization on in vivo release of norepinephrine in the bed nucleus of the stria terminalis in conscious rats. Brain Res 688:242-246.

Paschall GY, Davis M (2002) Olfactory-mediated fear-potentiated startle. Behav Neurosci 116:4-12.

Paxinos G, Watson C (1997) The rat brain in stereotaxic coordinates. San Diego: Academic.

Perrot-Sinal T, Ossenkopp KP, Kavaliers M (2000) Influence of a natural stressor (predator odor) on locomotor activity in the meadow vole (Mi- 
crotus pennsylvanicus): modulation by sex, reproductive condition, and gonadal hormones. Psychoneuroendocrinology 25:259-276.

Richardson R, Vishney A, Lee J (1999) Conditioned odor potentiation in rats. Behav Neurosci 113:787-794

Shipley MT, McLean JH, Ennis M (1995) Olfactory system. In: The rat nervous system (Paxinos G, ed), pp 899-926. San Diego: Academic.

Uwano T, Nishijo H, Ono T, Tamura R (1995) Neuronal responsiveness to various sensory stimuli, and associative learning in the rat amygdala. Neuroscience 68:339-361.

Vasilieva NY, Cherepanova EV, von Holst D, Apfelbach R (2000) Predator odour and its impact on male fertility and reproduction in Phodopus campbelli hamsters. Naturwissenschaften 87:312-314.

Vernet-Maury E, Polak EH, Demael A (1984) Structure-activity relationship of stress-inducing odorants in the rat. J Chem Ecol 10:1007-1018.

Walker DL, Davis M (1997) Double dissociation between the involvement of the bed nucleus of the stria terminalis and the central nucleus of the amygdala in startle increases produced by conditioned versus unconditioned fear. J Neurosci 17:9375-9383.

Walker DL, Davis M (2002) The role of amygdala glutamate receptors in fear learning, fear-potentiated startle, and extinction. Pharmacol Biochem Behav 71:379-392.

Wallace KJ, Rosen JB (2000) Predator odor as an unconditioned fear stimulus in rats: elicitation of freezing by trimethylthiazoline, a component of fox feces. Behav Neurosci 114:912-922.

Wallace KJ, Rosen JB (2001) Neurotoxic lesions of the lateral nucleus of the amygdala decrease conditioned fear but not unconditioned fear of a predator odor: comparison with electrolytic lesions. J Neurosci 21:3619-3627.

Zangrossi Jr H, File SE (1992) Behavioral consequences in animal tests of anxiety and exploration of exposure to cat odor. Brain Res Bull 29: $381-388$ 